Real i zat $i$ on of Root Rai sed Cosi ne Rol I- Of $f$ Filters Usi ng a Recursi ve FIR Filter Struct ure

\begin{tabular}{|c|c|}
\hline 著者 & Mukumot o Kai j i, Wada Tadahi r o \\
\hline $\begin{array}{l}\text { jour nal or } \\
\text { publ i cat i on title }\end{array}$ & I EEE Tr ansact i ons on Communi cat i ons \\
\hline vol une & 62 \\
\hline nunber & 7 \\
\hline page $r$ ange & $2456-2464$ \\
\hline year & $2014-06-09$ \\
\hline 出版者 & I EEE Communi cat i ons Soci et y \\
\hline 権利 & $\begin{array}{l}\text { ( C) } 2014 \text { I EEE. Per sonal use of thi s mat er i al } \\
\text { i s per mit t ed. Per mi ssi on from I EEE mist be } \\
\text { obt ai ned for al I ot her user s, i ncl udi ng } \\
\text { r epri nt i ng/ republ i shi ng thi s mat er i al for } \\
\text { advert i si ng or promot i onal pur poses, cr eat i ng } \\
\text { new col l ect i ve works for resal e or } \\
\text { r edi stri but i on to server s or I i st s, or reuse } \\
\text { of any copyr i ght ed component s of thi s work in } \\
\text { ot her works. }\end{array}$ \\
\hline URL & ht t p: //hdl . handl e. net /10297/7912 \\
\hline
\end{tabular}




\title{
Realization of Root Raised Cosine Roll-Off Filters Using Recursive FIR Filter Structure
}

\author{
Kaiji Mukumoto, Nonmember, Tadahiro Wada, Member, IEEE
}

\begin{abstract}
Root raised cosine roll-off (RRC) filter is one of the most fundamental elements in modern digital communications. This paper proposes a new method to efficiently realize the RRC filter. The method employs cubic Hermite interpolation to obtain a piecewise-polynomial approximation for the impulse response of the RRC filter. Then, the approximated impulse response is efficiently realized by using a transversal filter with a very small number of taps followed by integrators with simple recursive structure, named cascaded recursive finite impulse response (CRFIR) filter. As an example, an RRC filter with roll-off factor 0.5 is constructed by using the CR-FIR filter with only nine multipliers. Performance of the RRC filter in matched filtering is examined by computer simulations. Then, it is shown that the constructed RRC matched filter has nearly the same performance as the ideal filter.
\end{abstract}

Index Terms-Root raised cosine roll-off filter, recursive FIR Filter, Hermite interpolation, piecewise-polynomial approximation.

\section{INTRODUCTION}

$\mathbf{E}$ FFICIENT realization of pulse shaping filters and matched filters is one of the most elemental issues in modern digital communications such as software radios [1]. Pulse shaping filters are used for limiting the bandwidth of transmitting signals in transmitters. Matched filters are used for maximizing signal to noise ratio (SNR) in receivers. The combined impulse response of the pulse shaping and matched filters is required to satisfy the Nyquist criterion to avoid inter-symbol interference (ISI). It is well known that using a pair of root raised cosine roll-off (RRC) filters with the same roll-off factor meets the requirement in additive white noise environment [2].

Truncated finite impulse response (FIR) filters are commonly used to approximately realize the RRC filter since they can yield linear phase characteristics [3], [4]. However, conventional direct-form realization of the FIR filter requires a large number of multipliers and adders, which results in increasing the system costs such as processing time, power consumption and memory requirements. This problem is more serious in matched filtering since the input to the matched filter is signals with high frequency components requiring high sampling rate.

In this paper, we propose a novel method to efficiently realize truncated RRC impulse response by using recursive FIR filter structure. The method employs a piecewise-polynomial

K.Mukumoto is with the Division of Technical Service, Shizuoka University, Johoku 3-5-1, Naka-ku, Hamamatsu, 432-8561 Japan (e-mail: tekmuku@ipc.shizuoka.ac.jp).

T.Wada is with the Graduate School of Engineering, Shizuoka University, Johoku 3-5-1, Naka-ku, Hamamatsu, 432-8561, Japan. approximation for the impulse response of the RRC filter. Then, the approximated impulse response is realized by using a transversal filter with a very small number of taps followed by integrators with simple recursive structures. In this paper , we refer to this type of recursive FIR filters as cascaded recursive FIR (CR-FIR) filters.

Different types of recursive FIR filters have been proposed for various applications. For example, E. B. Hogenauer proposed a recursive FIR filter structure called cascaded integrator comb (CIC) filter for interpolation and decimation [5]. S. Chu and S.Burrus studied a class of recursive FIR filters which can efficiently realize piecewise-polynomial impulse responses by using a series of recursive equations [6]. R.Lehto et al. proposed another recursive FIR filter structure where the desired impulse response is represented by overlapped subpolynomials and realized by feedback loops with truncation capability [7], [8].

The latter two filters and the CR-FIR filter can exactly realize an arbitrary impulse response expressed by a piecewisepolynomial with integer coefficients. Hence, their performances are the same in the sense that they can realize the filter with the same impulse response. Moreover, these filters have almost the same efficiency, i.e., they can construct the same number of multipliers in the optimized implementation. However, different from the other two filters, the CR-FIR filter consists of completely separated sections: transversal part and integrator part, which is the same structure as the CIC filter except that the transversal part in the CIC filter is restricted to the form of comb filters.

Recursive FIR filters have so far been applied to conventional low pass filters (LPF), interpolation/decimation filters, audio equalizers, and raised cosine roll-off (RC) filters, etc. but not to RRC filters. In many practical cases, RRC filters are commonly realized by direct-form FIR filters truncated by using rectangular or other window functions [1]. Distributed arithmetic (DA) algorithm studied in [9] is also used to realize the RRC filters. A method to directly synthesis the waveform proposed in [10] is very efficient way to generate RRC pulses, although it can not be used for matched filtering. Moreover, studies on the realization of root Nyquist filters other than the $\mathrm{RRC}$ filters have been reported in a large number of papers, e.g. [3], [4], [11]-[13].

In this paper, we focus on the RRC filter, the most popular root Nyquist filter. We then develop a method to realize the RRC filter by using the CR-FIR filter structure. In order to construct the CR-FIR filter, the impulse response needs to be expressed by a piecewise-polynomial. We use Hermite interpolation [14] to derive the piecewise-polynomial approximating 


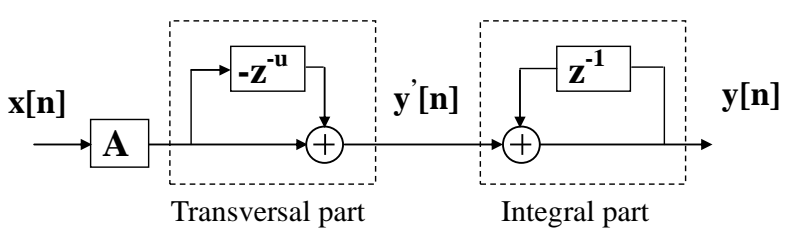

Fig. 1. Cascaded realization of the moving average filter.

the RRC impulse response. The polynomial approximation using Hermite interpolation is not optimum in the sense of minimizing approximation error. However, it is simple and sufficiently accurate for making the matched filter by using the CR-FIR filter structure. It is shown by computer simulations that the constructed CR-FIR filter with the piecewisepolynomial impulse response has enough performance as the RRC matched filter in spite of the fact that the number of multipliers in the filter is very small.

The rest of the paper is organized as follows: We first present a method to construct the CR-FIR filter in Section II. In Section III, we derive a piecewise-polynomial approximation to the impulse response of the RRC filter by using Hermite interpolation. In Section IV, it is shown by computer simulations that the CR-FIR filter with the piecewise-polynomial impulse response has enough performance as the RRC matched filter. Conclusions are remarked in Section V.

\section{CR-FIR FILTER}

\section{A. Basic idea}

To explain the basic idea of the CR-FIR filter, we begin with examining a moving average filter with the impulse response:

$$
h[n]=\left\{\begin{array}{ll}
A & 0 \leq n<u \\
0 & \text { otherwise }
\end{array},\right.
$$

where $A$ is a constant representing the filter gain and $u$ is the number of averaging samples. As is well known, this filter is efficiently realized (as a recursive FIR filter) by using a recursive equation:

$$
y[n]=y[n-1]+\dot{A(x}[n]-x[n-u]),
$$

where $x[]$ and $y[$ ] represent input and output of the filter, respectively. S.Chu and C.S. Burrus [6] generalized this idea to realize filters with an arbitrary impulse response represented by a piecewise-polynomial with integer coefficients. i.e., they realize the piecewise-polynomial impulse response by using a series of recursive equations.

On the other hand, the transfer function of the moving average filter, $H(z)$, is expressed as

$$
H(z)=\frac{\dot{A\left(1-z^{-u}\right)}}{1-z},
$$

and this can be realized as a cascade of a transversal filter and an integrator as shown in Fig.1. It is worth noting here that the CIC filters [5] used widely in interpolation/decimation are derived by multiplying this cascaded structure.

In this paper, we generalize the structure in Fig. 1 as follows: We first recognize that the impulse response of the transversal part is the derivative of the desired impulse response. Then,

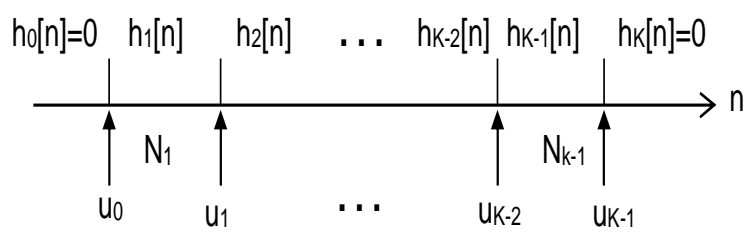

Fig. 2. Approximation intervals for the piecewise-polynomial.

by generalizing the number of derivations, we derive the CRFIR filter which can efficiently realize any impulse response expressed by a piecewise-polynomial with integer coefficients. Specifically, if the desired impulse response is expressed by a piecewise-polynomial with degree of at most $M$, it can be realized by cascading a transversal filter with the impulse response given by the $(M+1)$-th derivative of the desired impulse response and $(M+1)$ stages of integrators. Note that the transversal filter can be realized by using a small number of taps since the $(M+1)$-th derivative of the piecewisepolynomial becomes always zero except near the segment boundaries. In the following, we describe the method to construct this CR-FIR filter in detail.

\section{B. Filter construction}

Let us assume that a discrete time impulse response $h[n]$ of a desired filter is defined by a piecewise-polynomial with $(K+1)$ intervals as shown in Fig. 2, i.e., $h[n]$ is given by

$$
h[n]=\left\{\begin{array}{ll}
h_{0}[n]=0 & n<u_{0} \\
h_{k}[n] & u_{k-1} \leq n<u_{k} \text { for } k=1 \text { to } K-1 . \\
h_{K}[n]=0 & u_{K-1} \leq n
\end{array} .\right.
$$

We also assume for any $k$ that the impulse response $h_{k}[n]$ in $k$-th interval is expressed by a polynomial with degree of at most $M$.

When signal $x[n]$ is input to the filter, the output $y[n]$ is obtained by a convolution:

$$
y[n]=\sum_{i=u_{0}}^{u_{K-1}-1} h[i] x[n-i] .
$$

The number of product operations necessary to calculate this equation is $u_{K-1}-u_{0}$ times per each sample. However, by using the CR-FIR filter structure explained in the followings, we can significantly reduce the number of operations.

Let us define $m$-th order difference by

$$
h^{(m)}[n]=h^{(m-1)}[n]-h^{(m-1)}[n-1]
$$

and $h^{(0)}[n]=h[n]$. Furthermore, we define

$$
y^{(m)}[n]=\sum_{i=-\infty}^{\infty} h^{(m)}[i] x[n-i]
$$

and $y^{(0)}[n]=y[n]$. Note that $y^{(m)}[n]$ defined by (7) represents $m$-th order difference of $y[n]$ since substituting (6) into (7) yields

$$
y^{(m)}[n]=y^{(m-1)}[n]-y^{(m-1)}[n-1] .
$$


As we have assumed that $h_{k}[n]$ is a polynomial with degree of at most $M$, if the length of the interval, $N_{k}=u_{k}-u_{k-1}$, is greater than $M$,

$$
h^{(M+1)}[n]=h_{k}^{(M+1)}[n]=0
$$

holds for $u_{k-1}+M+1 \leq n<u_{k}(k=1,2, \cdots, K)$, where $u_{K}=\infty$. Moreover, it is obvious for $n<u_{0}$ that

$$
h^{(M+1)}[n]=h_{0}^{(M+1)}[n]=0 .
$$

Therefore, $(M+1)$-th order difference of $y[n]$ can be obtained as follows:

$$
\begin{aligned}
y^{(M+1)}[n] & =\sum_{i=-\infty}^{\infty} h^{(M+1)}[i] x[n-i] \\
& =\sum_{k=1}^{K} \sum_{i=u_{k-1}}^{u_{k-1}+M} h^{(M+1)}[i] x[n-i] \\
& =\sum_{k=1}^{K} \sum_{i=0}^{M} h^{(M+1)}\left[i+u_{k-1}\right] x\left[n-i-u_{k-1}\right] .
\end{aligned}
$$

From the obtained $y^{(M+1)}[n]$, we can get the filter output $y[n]=y^{(0)}[n]$ by applying the recursive equation derived from (8):

$$
y^{(m-1)}[n]=y^{(m-1)}[n-1]+y^{(m)}[n],
$$

repeatedly for $m=M+1, M, \cdots, 1$.

The number of multiplications necessary for this CR-FIR filter is only $(M+1) K$ times per sample, which is needed to calculate (11).

\section{Simplification in continuous impulse response}

If adjacent piecewise impulse responses $h_{k-1}[n]$ and $h_{k}[n]$ have the same value at their boundary, i.e.,

$$
h_{k-1}\left[u_{k-1}\right]=h_{k}\left[u_{k-1}\right]
$$

then

$$
h^{(M+1)}\left[u_{k-1}\right]=0
$$

holds as is shown in Appendix A. Therefore, by omitting the case of $i=0,(11)$ can be simplified to

$$
y^{(M+1)}[n]=\sum_{k=1}^{K} \sum_{i=1}^{M} h^{(M+1)}\left[i+u_{k-1}\right] x\left[n-i-u_{k-1}\right] .
$$

\section{Simplification in symmetric impulse response}

Linear phase filters require symmetric impulse responses, so that the case of symmetric impulse response is particularly important in practice. If the discrete time impulse response $h[n]$ is symmetric, (15) can further be simplified as is shown in Appendix B. i.e.,

$$
\begin{aligned}
y^{(M+1)}[n]= & \sum_{i=1}^{M} \sum_{k=1}^{K / 2} h^{(M+1)}\left[i+u_{k-1}\right]\left\{x\left[n-i-u_{k-1}\right]\right. \\
& \left.+(-1)^{(M+1)} x\left[n-M+i-u_{K-k}\right]\right\}
\end{aligned}
$$

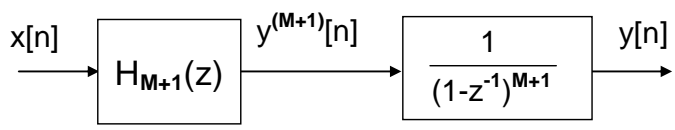

Fig. 3. Structure of the CR-FIR filter.

for the case that $K$ is even, and

$$
\begin{aligned}
y^{(M+1)}[n] & =\sum_{i=1}^{M}\left[\sum _ { k = 1 } ^ { ( K - 1 ) / 2 } h ^ { ( M + 1 ) } [ i + u _ { k - 1 } ] \left\{x\left[n-i-u_{k-1}\right]\right.\right. \\
& \left.+(-1)^{(M+1)} x\left[n-M+i-u_{K-k}\right]\right\} \\
& \left.+h^{(M+1)}\left[i+u_{(K-1) / 2}\right] x\left[n-i-u_{(K-1) / 2}\right]\right]
\end{aligned}
$$

for the case that $K$ is odd.

Accordingly, we can reduce the number of multiplications per sample to $M K / 2$ times for the case that $K$ is even and $M(K+1) / 2$ times for the case that $\mathrm{K}$ is odd.

\section{E. Issues in implementation}

For a causal filter, $z$-transform functions of the discrete time impulse response $h[n]$ and its $m$-th difference $h^{(m)}[n]$ are given by

$$
H(z)=\sum_{n=0}^{\infty} h[n] z^{-n}
$$

and

$$
H_{m}(z)=\sum_{n=0}^{\infty} h^{(m)}[n] z^{-n},
$$

respectively. Then, the relation

$$
H_{m}(z)=H(z)\left(1-z^{-1}\right)^{m}
$$

holds, so that, by substituting $m=M+1$, we can get

$$
H(z)=H_{M+1}(z) /\left(1-z^{-1}\right)^{M+1} .
$$

This equation implies that the transfer function $H(z)$ can be realized by cascading a filter with transfer function $H_{M+1}(z)$ and an $(M+1)$-th order integrator as shown in Fig.3. In this CR-FIR filter structure, the filter with transfer function $H_{M+1}(z)$ is realized by a non-recursive filter and it has a very small number of taps since the impulse response $h[n]$ is assumed to be a piecewise-polynomial with degree of at most $M$.

As is seen from (21), in order that the CR-FIR filter has truly finite impulse response, $H_{M+1}[z]$ must be perfectly divisible by $\left(1-z^{-1}\right)^{M+1}$. If all the coefficients of the piecewisepolynomial $h[n]$ are integer values, $H_{M+1}[z]$ satisfying the condition can be easily obtained by calculating (20) for $m=$ $M+1$.

In practice, however, the impulse response of a desired filter is rarely given as a piecewise-polynomial with integer coefficients. Thus, in order to realize a filter by using the CR-FIR filter structure, we first need to find an appropriate piecewisepolynomial with integer coefficients that can approximate the desired impulse response or its constant multiple. Furthermore, 
all operations must be performed in integer arithmetic so as to prevent the instability caused by rounding errors. Note that no roundoff error is commonly required in recursive FIR filters using integrators. This is because the integrator has a pole at $\mathrm{z}=1$ so that the output of the filter grows without bound if the pole is not exactly canceled.

Now, let the impulse response of a desired filter in continuous system be represented by $h_{o}(t)$. The discrete time impulse response $h_{o}[n]$ corresponding to $h_{o}(t)$ is given by $h_{o}[n]=h_{o}(n \Delta t) \Delta t$ when the sampling rate is $1 / \Delta t$. In order to realize the filter by using the CR-FIR filter structure, we first approximate $h_{o}(t)$ by a continuous time piecewise-polynomial $P_{h o}(t)$ with real-valued coefficeints. Then, $P_{h o}(t)$ is sampled at sampling rate $1 / \Delta t$ and multipled by an appropriate constant $K_{p}$ to obtain a discrete time piecewise-polynomial $P_{h}[n]$ with integer coefficients. Thus, the impluse response of the CR-FIR filter becomes $K_{p} / \Delta t$ times larger than that of the original filter, i.e.,

$$
P_{h}[n]=P_{h o}(n \Delta t) K_{p} \simeq h_{o}(n \Delta t) K_{p}=h_{o}[n] K_{p} / \Delta t .
$$

Therefore, in order to prevent the system overflow, the input $x$ to the CR-FIR filter is required to satisfy the condition:

$$
x<y_{\max } \Delta t /\left\{K_{p} \sum_{i=-\infty}^{\infty}\left|h_{o}(i \Delta t) \Delta t\right|\right\}
$$

where $y_{\max }$ represents the maximum number in the system. In other words, smaller $K_{p}$ is required to enlarge the input dynamic range.

\section{ROOT RAISED COSINE FILTER}

The impulse response of an RRC filter with roll-off factor $\alpha$ is given by

$h(t)=\frac{1}{1-(4 \alpha t)^{2}}\left[\frac{1}{\pi t} \sin ((1-\alpha) \pi t)+\frac{4 \alpha}{\pi} \cos ((1+\alpha) \pi t)\right]$,

where $t$ is normalized by the symbol period. Note that this equation has the following singular points:

$$
t=0 \text { and } t= \pm 1 /(4 \alpha) \text {. }
$$

The values at these points are given by

$$
h(0)=1-\alpha+4 \alpha / \pi
$$

and

$$
h\left( \pm \frac{1}{4 \alpha}\right)=\frac{1+\alpha}{2} \sin \left(\frac{(1+\alpha) \pi}{4 \alpha}\right)+\frac{2 \alpha}{\pi} \sin \left(\frac{(1-\alpha) \pi}{4 \alpha}\right),
$$

respectively.

In the followings, we will present a way to approximate (24) by a piecewise-polynomial. There are various kinds of methods to approximate a function by a polynomial. Among such methods, in this paper, we choose to employ cubic Hermete interpolation algorithm since it is simple and gives sufficient approximation for use in the CR-FIR filter.

\section{A. cubic Hermite polynomial approximation}

In general, cubic Hermite polynomial interpolation imposes conditions that the approximating polynomial and the approximated function have the same values and derivatives at the both boundary points of the approximation interval. That is, representing the approximated function as $f(x)$ and the approximation interval as $\left(x_{L}, x_{U}\right)$, we can obtain the coefficients of the approximating polynomial

$$
P(x)=a x^{3}+b x^{2}+c x+d
$$

from conditions

$$
\begin{gathered}
P\left(x_{L}\right)=f\left(x_{L}\right) \quad, \quad P\left(x_{U}\right)=f\left(x_{U}\right) \\
P^{\prime}\left(x_{L}\right)=f^{\prime}\left(x_{L}\right) \quad, \quad P^{\prime}\left(x_{U}\right)=f^{\prime}\left(x_{U}\right) .
\end{gathered}
$$

\section{B. Deciding approximation intervals}

The cubic Hermite polynomial interpolation uniquely determines an approximate polynomial when its approximation interval is decided. We thus consider in this section how to decide the best approximation intervals to approximate the impulse response $h(t)$ of RRC filter for an application to the matched filtering. Since $h(t)$ is symmetric with respect to $t=0$ and this is the constraint for the filter having linear phase response, it is reasonable to assume that an approximate polynomial $P_{h}(t)$ is also symmetric, i.e.,

$$
P_{h}(t)=P_{h}(-t)
$$

We thus consider only non-negative region. Moreover, for simplicity, we consider only the case that there are three approximation intervals in the non-negative region, i.e., six approximation intervals in all. Let us put the first boundary point at $t=0$ and denote the second and third points by $t_{1}$ and $t_{2}$, respectively. Then, the approximate polynomial $P_{h}(t)$ is expressed by

$$
P_{h}(t)= \begin{cases}P_{h 1}(t) & 0 \leq t<t_{1} \\ P_{h 2}(t) & t_{1} \leq t<t_{2} \\ P_{h 3}(t)=0 & t_{2} \leq t\end{cases}
$$

where $P_{h 1}(t), P_{h 2}(t)$, and $P_{h 3}(t)$ represent approximate polynomials for each region, respectively.

At $t=0$, it is derived from (24) and (26) that

$$
h(0)=1-\alpha+4 \alpha / \pi
$$

and

$$
h^{\prime}(0)=0 \text {. }
$$

For deciding $t_{2}$, we begin by assuming that $P_{h}(t)$ is continuous at $t_{2}$. By this assumption, $P_{h 2}\left(t_{2}\right)$ must be 0 since $P_{h}(t)=P_{h 3}(t)=0$ for $t \geq t_{2}$. Thus, $t_{2}$ is selected from the points satisfying

$$
h(t)=0 .
$$

Among those points, in this paper, we choose the second smallest positive point as $t_{2}$ so that the curve of $h(t)$ in region $\left(t_{1}, t_{2}\right)$ can be sufficiently approximated by a cubic polynomial. 
To determine $t_{1}$, let us consider the case that an RRC pulse $p_{t}(t)$ expressed by the same equation as $h(t)$, namely (24), is input to the filter. Then, the output is given by

$$
y(t)=\int_{-\infty}^{\infty} P_{h}(\lambda) p_{t}(t-\lambda) d \lambda .
$$

Since we are assuming that the filter is applied to matched filtering, $t_{1}$ should be determined so that the filter output (35) has minimum ISI at the Nyquist instants. For easily find such a point, we ignore the case of $t>2$ and adopt an evaluation function:

$$
J=\left\{y^{2}(1)+y^{2}(2)\right\} / y^{2}(0) .
$$

Then, $t_{1}$ is decided as the point to minimize (36) by using numerical calculation.

In practice, $t_{1}$ and $t_{2}$ are needed to be set to integer multiples of the sampling period. Moreover, to avoid instability caused by rounding errors, coefficients of the approximate polynomial are required to be integer values. Therefore, $t_{1}, t_{2}$, and the coefficients have to be adjusted to meet the conditions in filter designing.

\section{Example}

As an example, we here consider an RRC matched filter with roll-off factor $\alpha=0.5$ and sampling period $\Delta t=0.05$. For the case of $\alpha=0.5$, although the second smallest positive point among the points satisfying (34) is $1.69 \cdots$, we choose $t_{2}=1.7$ so that it becomes integer multiples of $\Delta t$. On the other hand, in order to determine $t_{1}$, the point minimizing (36) is obtained as $0.87 \cdots$ by numerical calculation. Then, we choose $t_{1}=0.85$, the nearest value among integer multiples of $\Delta t$.

For these intervals, the cubic Hermite polynomial approximation yields

$$
P_{h}(t)= \begin{cases}P_{h 1}(t) & |t|<0.85 \\ P_{h 2}(t) & 0.85 \leq|t|<1.7 \\ P_{h 3}(t)=0 & 1.7 \leq|t|\end{cases}
$$

where

$$
\begin{aligned}
& P_{h 1}(t)=2.03|t|^{3}-3.26|t|^{2}+0|t|+1.137 \\
& P_{h 2}(t)=-1.08|t|^{3}+4.97|t|^{2}-7.26|t|+3.27 .
\end{aligned}
$$

In order to use the polynomial as the impulse response of the CR-FIR filter, we first discretize the time with $\Delta t$, and then quantize the coefficients of $P_{h 1}(t)$ and $P_{h 2}(t)$ after multiplying an appropriate constant. We choose the constant $K_{p}=(1 / \Delta t)^{3} / 1.015$ so that the coefficients of the highest order terms in (38) and (39) are to be small integers. Then, we get

$$
K_{p} \cdot P_{h}(n \Delta t) \cong P_{h}[n]= \begin{cases}P_{h 1}[n] & |n|<17 \\ P_{h 2}[n] & 17 \leq|n|<34 \\ P_{h 3}[n]=0 & 34 \leq|n|\end{cases}
$$

where

$$
\begin{aligned}
& P_{h 1}[n]=2|n|^{3}-64|n|^{2}+0|n|+8958 \\
& P_{h 2}[n]=-|n|^{3}+98|n|^{2}-2862|n|+25771 .
\end{aligned}
$$

However, these polynomials do not satisfy the conditions to simplify the CR-FIR filter structure, i.e., the continuous conditions:

$$
P_{h 1}\left[t_{1} / \Delta t\right]=P_{h 2}\left[t_{1} / \Delta t\right]
$$

and

$$
P_{h 2}\left[t_{2} / \Delta t\right]=0 \text {. }
$$

We thus adjust the polynomials as follows: The coefficients of $|n|^{3}$ are fixed at the values in (41) and (42), respectively. The coefficient of $|n|$ in (41) is also fixed at 0 to smoothly connect the polynomials at $n=0$. The other coefficients are adjusted so as to satisfy the conditions of (43), (44), and another additional condition:

$$
P_{h 1}^{\prime}\left[t_{1} / \Delta t\right]=P_{h 2}^{\prime}\left[t_{1} / \Delta t\right]
$$

for smoothing the connection between the polynomials (41) and (42). Among the piecewise-polynomials with the integer coefficients satisfying the conditions, we search the one that minimizes the evaluation function (36) by using numerical calculation. The exact way to calculate $y()$ in (36) is explained in Section IV. By this operation, we obtain the following adjusted piecewise-polynomial:

$$
P_{a h}[n]= \begin{cases}P_{a h 1}[n] & |n|<17 \\ P_{a h 2}[n] & 17 \leq|n|<34 \\ P_{a h 3}[n]=0 & 34 \leq|n|\end{cases}
$$

where

$$
\begin{aligned}
& P_{a h 1}[n]=2|n|^{3}-62|n|^{2}+0|n|+8092 \\
& P_{a h 2}[n]=-|n|^{3}+90|n|^{2}-2567|n|+22542 .
\end{aligned}
$$

In Fig.4, the normalized form of this approximate piecewisepolynomial given by

$$
\tilde{P}_{a h}(n \Delta t)=\tilde{P}_{a h}[n]=P_{a h}[n] / \sqrt{\sum_{i=-34}^{34} P_{a h}^{2}[i] \Delta t .}
$$

is shown by a solid line together with the theoretical RRC impulse response (24) depicted by a dashed line. We see from the figure that the approximate piecewise-polynomial is almost the same as the theoretical RRC impulse response in the approximation interval $(-1.7,1.7)$.

\section{IMPLEMENTATION}

By shifting $P_{a h}[n]$ by 34 samples, we can obtain a causal impulse response $P_{c}[n]$ represented by

$$
P_{c}[n]= \begin{cases}P_{c 1}[n] & |n-34| \leq 17 \\ P_{c 2}[n] & 17 \leq|n-34| \leq 34 \\ 0 & 34 \leq|n-34|\end{cases}
$$

where

$$
\begin{aligned}
& P_{c 1}[n]=2|n-34|^{3}-62|n-34|^{2}+8092, \\
& P_{c 2}[n]=-|n-34|^{3}+90|n-34|^{2}-2567|n-34|+22542 .
\end{aligned}
$$

The number of intervals for the piecewise-polynomial approximation is $K+1=6$, so that $K$ is an odd number. Then, setting the boundary points as $u_{0}=0, u_{1}=17, u_{2}=34, u_{3}=52$, $u_{4}=69, P_{c}[n]$ is symmetric with respect to $n=u_{2}$ and 


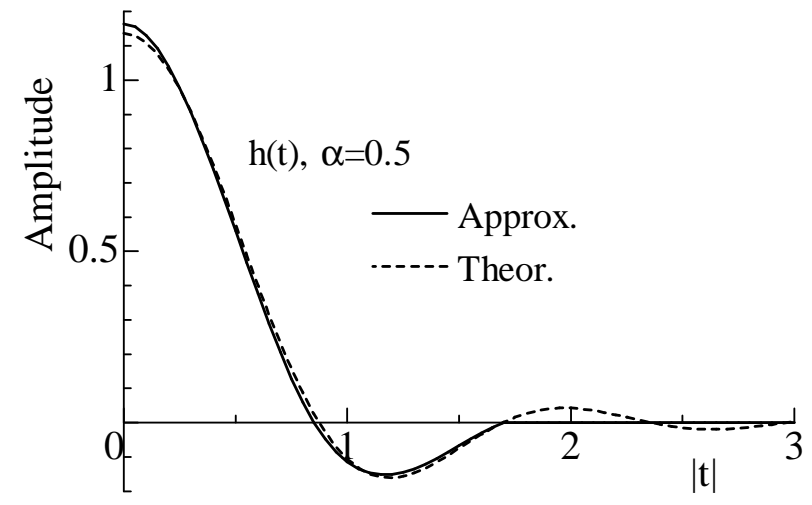

Fig. 4. Comparison with the piecewise-polynomial and the RRC impulse response.

continuous at $u_{k-1}(k=1,2,3)$. Thus, (17) can be applied to obtain $y^{(4)}[n]$. The coefficients needed to calculate $y^{(4)}[n]$ are $P_{c}^{(4)}\left[i+u_{k-1}\right](k=1,2,3 ; i=1,2,3)$ and they are obtained from (6) as

$$
\begin{array}{lll}
P_{c}^{(4)}[1]=-96, & P_{c}^{(4)}[2]=174, & P_{c}^{(4)}[3]=-72, \\
P_{c}^{(4)}[18]=-2, & P_{c}^{(4)}[19]=-12, & P_{c}^{(4)}[20]=-4, \\
P_{c}^{(4)}[35]=4, & P_{c}^{(4)}[36]=16, & P_{c}^{(4)}[37]=4 .
\end{array}
$$

Output of the filter, $y^{(0)}[n]$, is obtained by integrating $y^{(4)}[n]$ four times by using the recursive equation (12).

\section{PERFORMANCE EVALUATION}

The frequency response of the constructed CR-FIR filter is depicted by a solid line in Fig.5, where the $x$-axis represents the frequency normalized by the symbol rate. For the sake of comparison, the frequency responses of the ideal RRC filter and truncated FIR (TR-FIR) filter with rectangular window of the length 69 samples (the same response length as the CR-FIR filter) are also shown by a dashed line and a dotdashed line, respectively. All the three filters are designed for matched filtering the RRC pulse with roll off factor 0.5. We see from the figure that the CR-FIR filter has better stop band attenuation (i.e., in the sense that it has smaller side lobes until the third one) than the TR-FIR filter although it is designed to reduce the ISI. This is because the piecewise-polynomial impulse response in the CR-FIR filter, derived by Hermite polynomial approximation, is smoothly connected.

Next, we consider the ISI performance of the CR-FIR filter in the matched filtering. For that purpose, an ideal RRC pulse $p_{t}(t)$, which is equal to $h(t)$ in (24), is assumed to be fed to the filter. Then, let us denote the input and output signals to the filter in the discrete time system by $p_{t}[n]$ and $y[n]$, respectively. In this case, by noting that $p_{t}[n]$ and $y[n]$ respectively correspond to input $p_{t}(n \Delta t) \Delta t$ and output $y(n \Delta t) \Delta t$ in the continuous time system, we can get

$$
y(n \Delta t)=y[n] / \Delta t=\frac{1}{\Delta t} \sum_{i=-\infty}^{\infty} p_{t}[i] \tilde{P}_{h}[n-i],
$$

where $\tilde{P}_{h}[n]$ is the (non-causal) normalized impulse response defined in (49). This $y(n \Delta t)$ is used for calculating the

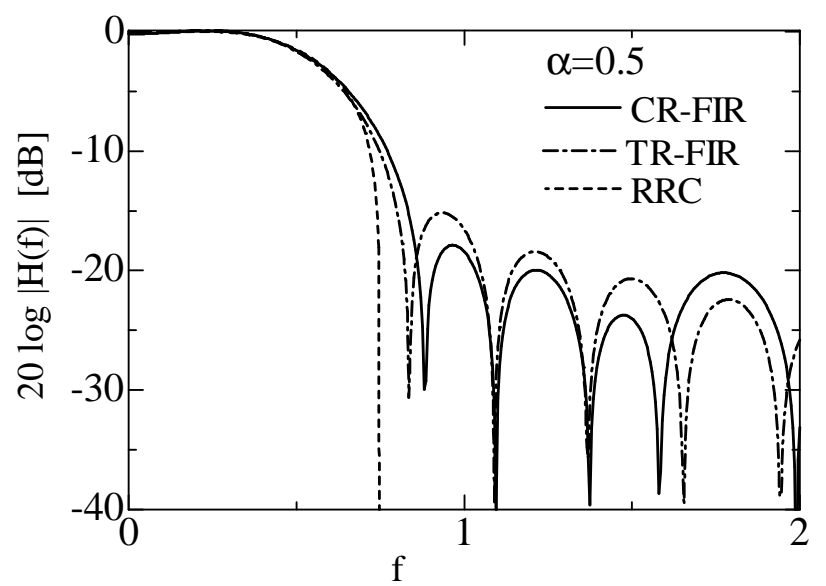

Fig. 5. Frequency response.

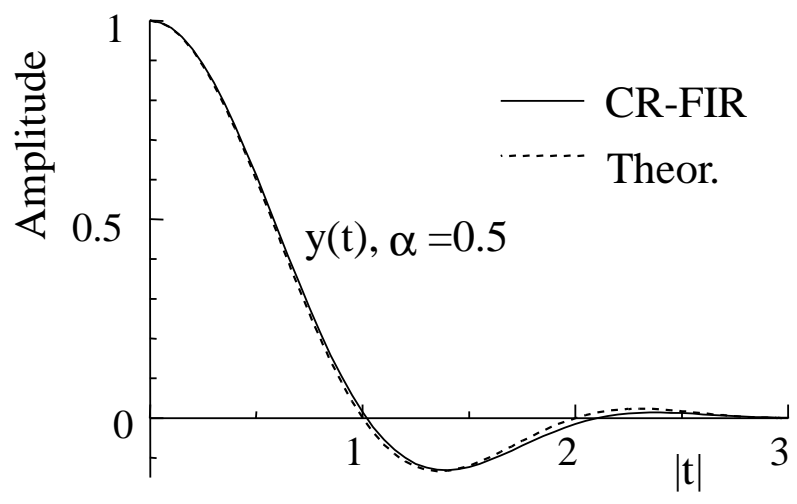

Fig. 6. Output of the matched filter.

evaluation function (36) to derive the adjusted piecewisepolynomial (46).

In Fig.6, the output signal $y(n \Delta t)$ from the CR-FIR filter is depicted by a solid line together with the theoretical RC pulse signal depicted by a dashed line, which represents the output signal from the ideal RRC filter. We see that both curves are very similar. Moreover, the curve with solid line also has 1 at $t=0$ and almost 0 at $|t|=1,2, \ldots$, meaning that the ISI is very small. In fact, the amount of ISI approximately evaluated by substituting (53) into (36) is 0.00019 . The output signal from the TR-FIR filter is not depicted in the figure since it is almost the same as the solid line. The amount of ISI for the TR-FIR filter evaluated by (36) is 0.00023 , a slightly larger than that of the CR-FIR filter.

Finally, we evaluate the performance of the CR-FIR filter in the classical quadrature amplitude modulation (QAM) systems [2] by computer simulations. In the QAM demodulator, perfect carrier synchronization is assumed, so that the in-phase and quadrature phase signals are independent series of RRC pulses with different levels. Those pulses are applied to the matched filter and the outputs of the each matched filter are assumed to be sampled at perfect timing to decide the received symbol. The average pulse energy per bit is denoted by $E_{b}$ and the additive white gaussian noise (AWGN) with one sided power 


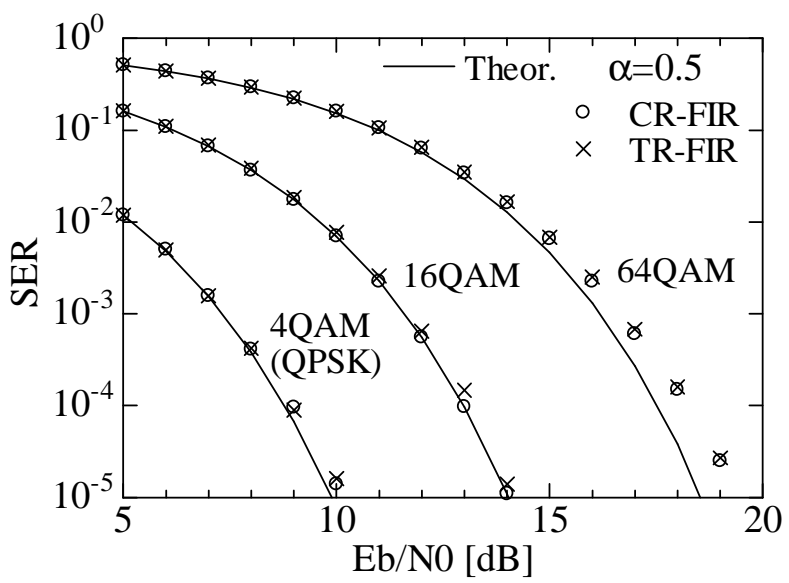

Fig. 7. SER performances of QAM demodulators.

spectral density $N_{0}$ is assumed.

In Fig.7, circles represent symbol error rates (SER) for the QAM demodulators (4QAM, 16QAM, and 64 QAM) with the in-phase and quadrature phase matched filters realized by using the CR-FIR filter structure explained in III-D. Crosses represent SER performances for the case that the TR-FIR filters are used as the matched filters. The curves with solid line represent the theoretical SER performances. As is seen from the figure, the circles and crosses exhibit almost the same performance in spite of the fact that the CR-FIR filter requires only 9 multiplications per sample whereas the TRFIR filter requires 69 multiplications per sample. Moreover, we see from the figure that both the simulation results exhibit nearly the same performance as the theoretical curves although they slightly degrade at higher $E_{b} / N_{0}$ in 64QAM.

In this section, we have demonstrated the performances of the CR-FIR filter by using a concrete example constructed in Section III. Then, it is shown that the CR-FIR filter realizing piecewise-polynomial impulse response derived by Hermite approximation has sufficient performances as the matched filters in QPSK and 16 QAM demodulators. Here it is worth noting that the performance of the CR-FIR filter is mostly determined by the realizing polynomial impulse response since the CR-FIR filter can exactly realize the polynomial impulse response with integer coefficients. This implies the possibility to further improve the performance by using more sophisticated approximation methods instead of the Hermite approximation. However, more easy way to improve the performance is increasing the number of intervals for the piecewise-polynomial since the Hermite approximation and the CR-FIR filter structure can be straightforwardly extended for realizing the piecewise-polynomial with lager number of intervals.

\section{CONCLUSION}

In this paper, we have proposed a novel method to very efficiently realize RRC filters by using recursive FIR filter structure. The method assumes that the impulse response of the filter is given by a piecewise-polynomial. Then, the filter is realized by using a transversal filter with a very small number of taps followed by integrators with simple recursive structures. We have referred to this type of recursive FIR filters as CR-FIR filters. The CR-FIR filter can further be simplified when the piecewise-polynomial is continuous and/or symmetric. To easily obtain a piecewise-polynomial that approximates an RRC impulse response, we have proposed to take advantage of Hermite interpolation. As an example, we have designed an RRC filter with roll-off factor 0.5. The RRC filter can be realized by a CR-FIR filter with only nine multipliers. Then, it is shown that the filter exhibits almost the same performance to the ideal RRC filter when it is used as matched filters in QPSK and 16QAM demodulators.

The advantage of recursive FIR filters such as the CR-FIR filter to other FIR filters is the lower arithmetic complexity. The number of required multipliers for the recursive FIR filters is proportional to the number of intervals for the piecewise-polynomial and the order of the polynomial rather than the actual filter order, so that the recursive FIR filters are particularly efficient for systems with high over sampling factor. The advantage of the CR-FIR filter to other recursive FIR filters is conceptual and structural simplicity. Due to the completely separated filter structure, we can easily obtain the filter tap gains only by calculating $(M+1)$-th order difference of the desired impulse response.

Although in this paper we have focused on applying the CR-FIR filter to matched filtering, it can clearly be used for pulse shaping as well. Discussions on applying the CR-FIR filter to pulse shaping are left for a future paper. Moreover, studies on more efficient implementation of the CR-FIR filers by taking the advantage of the completely cascaded structure are also left as a future work. Practical issues such as effects of analog front-end and influence of symbol timing jitter [15] are also remained for future works.

\section{APPENDIX A}

SIMPLIFICATION FOR CONTINUOUS IMPULSE RESPONSE

Here, we consider the case that

$$
h_{k-1}\left[u_{k-1}\right]=h_{k}\left[u_{k-1}\right]
$$

and $u_{k-1}-u_{k-2}>M+1$ where $u_{k-2}$ and $u_{k-1}$ are arbitrary adjacent boundary points. In this case, we can write

$$
h^{(M+1)}\left[u_{k-1}\right]=h_{k-1}^{(M)}\left[u_{k-1}\right]-h_{k-1}^{(M)}\left[u_{k-1}-1\right] .
$$

Then, by repeatedly substituting (6) for the first term, it becomes

$$
h^{(M+1)}\left[u_{k-1}\right]=h_{k-1}^{(0)}\left[u_{k-1}\right]-\sum_{m=0}^{M} h_{k-1}^{(m)}\left[u_{k-1}-1\right] .
$$

Since we can rewrite the second summation as

$$
\begin{aligned}
\sum_{m=0}^{M} h_{k-1}^{(m)}\left[u_{k-1}-1\right] & =\sum_{m=0}^{M}\left\{h_{k-1}^{(m)}\left[u_{k-1}\right]-h_{k-1}^{(m+1)}\left[u_{k-1}\right]\right\} \\
& =h_{k-1}^{(0)}\left[u_{k-1}\right]-h_{k-1}^{(M+1)}\left[u_{k-1}\right], \text { (A.4) }
\end{aligned}
$$

(A.3) becomes

$h^{(M+1)}\left[u_{k-1}\right]=h_{k-1}^{(0)}\left[u_{k-1}\right]-h_{k-1}^{(0)}\left[u_{k-1}\right]+h_{k-1}^{(M+1)}\left[u_{k-1}\right]$. 


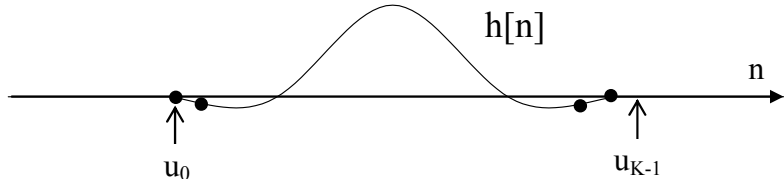

Fig. B.1. Symmetric impulse response.

The first term is canceled by the second term. Moreover, since we have assumed that $h_{k-1}[n]$ is a polynomial with degree of at most $M$ and $u_{k-1}-u_{k-2}>M+1$, the third term must be 0 . Therefore, we derive the result:

$$
h^{(M+1)}\left[u_{k-1}\right]=0 .
$$

\section{APPENDIX B}

\section{SIMPLIFICATION FOR SYMMETRIC IMPULSE RESPONSE}

Here, we consider the case that $h[n]$ is a symmetric impulse response and assume that the start point $u_{0}$ and the end point $u_{K-1}$ are located as shown in Fig. B.1. In this case, it holds the following equation:

$$
h\left[u_{0}+n\right]=h\left[u_{K-1}-1-n\right]
$$

from the assumption of symmetry. Then, by induction using this equation and (6), we can derive

$$
h^{(m)}\left[u_{0}+n\right]=(-1)^{m} h^{(m)}\left[u_{K-1}-1-n+m\right] .
$$

In the followings, let us consider separately the cases where $K$ is even or odd.

[The case that $K$ is even]

In the case that $K$ is an even number,

$$
u_{k-1}-u_{0}=u_{K-1}-u_{K-k}
$$

holds for any $k$. Thus, substituting this equation into $u_{k-1}$ of (11) and calculating the internal summation of (11) using (B.3), it becomes

$$
\begin{gathered}
\sum_{i=0}^{M} h^{(M+1)}\left[i+u_{k-1}\right] x\left[n-i-u_{k-1}\right] \\
=(-1)^{M+1} \sum_{i=0}^{M} h^{(M+1)}\left[i+u_{K-k}\right] x\left[n-M+i-u_{k-1}\right] .
\end{gathered}
$$

Using this equation in the calculation of the summation for $k>K / 2$, we can derive

$$
\begin{array}{r}
y^{(M+1)}[n]=\sum_{k=1}^{K / 2} \sum_{i=0}^{M} h^{(M+1)}\left[i+u_{k-1}\right] x\left[n-i-u_{k-1}\right] \\
+(-1)^{M+1} \sum_{k=K / 2+1}^{K} \sum_{i=0}^{M} h^{(M+1)}\left[i+u_{K-k}\right] x\left[n-M+i-u_{k-1}\right] .
\end{array}
$$

In the calculation of the second term of this equation, by changing the variable from $k$ to $K+1-h$ and then replacing $h$ with $k$ again, we can obtain

$$
\begin{aligned}
y^{(M+1)}[n]= & \sum_{k=1}^{K / 2} \sum_{i=0}^{M} h^{(M+1)}\left[i+u_{k-1}\right]\left\{x\left[n-i-u_{k-1}\right]\right. \\
& \left.+(-1)^{M+1} x\left[n-M+i-u_{K-k}\right]\right\} .
\end{aligned}
$$

Equation (16) can be derived from this equation by changing the order of the summations and omitting the case that $i=0$ since the impulse response in (16) is assumed to be continuous at $u_{k-1}(k=1,2, \cdots, K / 2)$.

[The case that $K$ is odd]

In the case that $K$ is an odd number, (B.3) holds for any $k$ except $k=(K-1) / 2+1$. Therefore, in the calculation of the outer summation in (11), we consider separately the cases where $k \leq(K-1) / 2, k=(K+1) / 2$, or $k \geq(K+3) / 2$. Then, substituting (B.4) for the summation of the case where $k \geq(K+3) / 2$, it becomes

$$
\begin{aligned}
& y^{(M+1)}[n]=\sum_{k=1}^{(K-1) / 2} \sum_{i=0}^{M} h^{(M+1)}\left[i+u_{k-1}\right] x\left[n-i-u_{k-1}\right] \\
& +(-1)^{M+1} \sum_{k=(K+3) / 2}^{K} \sum_{i=0}^{M} h^{(M+1)}\left[i+u_{K-k}\right] x\left[n-M+i-u_{k-1}\right] \\
& +\sum_{i=0}^{M} h^{(M+1)}\left[i+u_{(K-1) / 2}\right] x\left[n-i-u_{(k-1) / 2}\right] .
\end{aligned}
$$

Equation (17) can be derived from this equation by almost the same way as the case that $K$ is even, i.e., by replacing the variable, changing the order of the summations, and omitting the case that $i=0$ since the impulse response in (17) is assumed to be continuous at $u_{k-1}(k=1,2, \cdots,(K-1) / 2)$.

\section{REFERENCES}

[1] B. Farhang-Boroujeny, Signal Processing techniques for Software Radios, Lulu publishing house, 2008.

[2] A. B. Carlson, P. B. Crilly, and J. C. Rutledge, Communication Systems, 4th ed., McGraw-Hill, 2002.

[3] B. Farhang-Boroujeny, A square-root Nyquist (M) filter design for digital communication systems, IEEE Trans., Signal Proc., vol.56, pp.2127-2132, May 2008.

[4] K. Väisänen and M. Renfors, Efficient digital filters for pulse-shaping and jitter-free frequency error detection and timing recovery, Signal Processing, 81, pp.829-844, 2001.

[5] E. B. Hogenauer, An economical class of digital filters for decimation and interpolation, IEEE Trans. on Acoustics, Speech and Signal Processing, Vol.ASSP-29, pp.155-162, April 1981.

[6] S. Chu, S. Burrus, Efficient recursive realizations of FIR filters, Part I: The filter structures," Circ. Syst. Signal Process, Vol.3, No.1, pp.2-20 1984.

[7] R. Lehto, T. Tauren, O. Vainio, Recursive FIR filter structures on FPGA, Microprocessors and Microsystems, 35, pp.595-602 2011.

[8] O. Vainio and T. Saramäki, Piecewise-Polynomial Approximation for Raised-Cosine Filters, 5-th Nordic Signal Processing Symposium(NORSIG-2002), Oct. 2002.

[9] I. Hatai and I. Chakrabarti, Multi-standard programmable baseband modulator for next generation wireless communication, IJCNC, vol.2, No.4, pp.58-71, July 2010.

[10] M. L. Welborn, Direct waveform synthesis for software radios, WCNC, vol.1, pp.211-215, 1999.

[11] R. Mehra and S. Devi, Area efficient \& cost effective pulse shaping filter for software radios, IJASUC, Vol.1, No.3 pp.85-91, Sep. 2010.

[12] X. Zhang and H. Iwakura, Design of IIR Nyquist filters with zero intersymbol interference, IEICE, Trans. vol. E79-A, No.8, pp.1139-1144, Aug 1996.

[13] A. Eghbali, T. Saramäki, and H. Johansson, On two-stage Nyquist pulse shaping filters, IEEE Trans. Signal Processing, vol.60, no.1, pp.483-488, Jan. 2012.

[14] K. E. Atkinson, An introduction to numerical analysis 2nd ed., John Wiley\&Sons, 1989

[15] Chia-Yu Yao, 'The Design of Hybrid Asymmetric-FIR/Analog PulseShaping Filters Against Receiver Timing Jitter," IEEE Trans. Commun., vol.60, no.5, pp.1199-1203, May 2012. 
Kaiji Mukumoto received the Ph.D. degree in electrical engineering from Shizuoka University, Hamamatsu, Japan, in 1993. He is currently with the Division of Technical Service, Shizuoka University,

PLACE PHOTO HERE as a technical specialist. His current research interest includes communication theory, software radios, wireless sensor networks, and meteor burst communications.

\begin{tabular}{|l|} 
\\
\\
PLACE \\
PHOTO \\
HERE \\
\end{tabular}

Tadahiro Wada (S'96 -M'98) received the B.E. and M.E. and $\mathrm{Ph}$. D. degrees in engineering from Nagoya University, Japan, in 1993, 1995, and 1998, respectively.

Since 1998, he has been with the Faculty of Engineering, Shizuoka University, Hamamatsu, Japan, where he was firstly an assistant professor, and is currently an associate processor. From 2004 to 2005 , he was a visiting researcher of the University of Sydney, Australia.

His current research interests are cooperative networks, communication theory, error control techniques, and meteor burst communications. He received the Best Tutorial Paper Award from IEEE ComSoc in 2006. 